Journal of Applied AnALysis

Vol. 8, No. 2 (2002), pp. 261-278

\title{
OSCILLATION OF SOLUTIONS TO NONLINEAR NEUTRAL DELAY DIFFERENTIAL EQUATIONS
}

\author{
S. H. SAKER and I. KUBIACZYK \\ Received August 28, 2001 and, in revised form, May 15, 2002
}

\begin{abstract}
In this paper we shall consider the nonlinear neutral delay differential equations with variable coefficients. Some new sufficient conditions for oscillation of all solutions are obtained. Our results extend and improve some of the well known results in the literature. Some examples are considered to illustrate our main results. The neutral logistic equation with variable coefficients is considered to give some new sufficient conditions for oscillation of all positive solutions about its positive steady state.
\end{abstract}

\section{Introduction}

In recent years the literature on the oscillation theory of neutral delay differential equations is growing very fast. It is relatively a new field with interesting applications in real world life problems. In fact, the neutral delay differential equations appear in modelling of the networks containing lossless transmission lines (as in high-speed computers where the lossless transmission lines are used to interconnect switching circuits), in the study

2000 Mathematics Subject Classification. 34K11, 34K40.

Key words and phrases. Oscillation, nonlinear neutral delay differential equations, neutral delay logistic equation.

ISSN 1425-6908 (C) Heldermann Verlag. 
of vibrating masses attached to an elastic bar, as the Euler equation in some variational problems, theory of automatic control and in neuromechanical systems in which inertia plays an important role. see Hale [16], Driver [8], Brayton and Willoughby [5], Popov [23] and Boe and Chang [4] and reference cited therein).

Recently some papers $[13,34]$ have appeared which are concerned with the oscillation and nonoscillation behavior of the neutral delay differential equation with variable coefficients,

$$
\frac{d}{d t}[a(t) x(t)-P(t) x(t-\tau)]+Q(t) f(x(t-\sigma))=0, \quad t \geq t_{0}
$$

where

$$
a, P, Q \in C\left(\left[t_{0}, \infty\right), \mathbb{R}^{+}\right), \quad \sigma, \tau \in[0, \infty),
$$

and $f$ satisfies

$$
\begin{aligned}
& f \in C\left(\left[t_{0}, \infty\right), \mathbb{R}\right), u f(u)>0 \text { for } u \neq 0 \\
& \text { and } \lim _{u \rightarrow 0} \frac{u}{f(u)}=\beta \text { exists. }
\end{aligned}
$$

Let $\rho=\max \{\sigma, \tau\}$ and let $t_{1} \geq t_{0}$. By a solution of equation (1.1) on $\left[t_{1}, \infty\right)$ we mean a function $x \in C\left(\left[t_{1}-\rho, \infty\right), \mathbb{R}\right)$, such that $(a(t) x(t)-P(t) x(t-\tau))$ is continuously differentiable on $\left[t_{1}, \infty\right)$ and such that equation (1.1) is satisfied for $t \geq t_{1}$.

Let $t_{1} \geq t_{0}$ be a given point, let $\phi \in C\left(\left[t_{1}-\rho, t_{1}\right], \mathbb{R}\right)$ be a given initial function. By using the method of steps one can see that equation (1.1) has a unique solution $x \in C\left(\left[t_{1}-\rho, \infty\right), \mathbb{R}\right)$ such that

$$
x(t)=\phi(t) \quad \text { for } t \in\left[t_{1}-\rho, t_{1}\right] .
$$

As usual, we say that the equation (1.1) is oscillatory if every solution of (1.1) is oscillatory, i.e., for every initial point $t_{1} \geq t_{0}$ and for every initial function $\phi \in C\left(\left[t_{1}-\rho, t_{1}\right], \mathbb{R}\right)$ the unique solution of equations (1.1) and (1.4) has arbitrarily large zeros. Otherwise the solution is called nonoscillatory. The oscillation of various functional differential equations has been investigated by several authors. For some contributions we refer to the monographs $[1,2,3,9,15,21]$.

The first systematic work about oscillation of neutral delay differential equations is given by Zahariev and Bainov [32]. For the oscillation of equation (1.1) when $P(t)$ and $Q(t)$ are constants, $a(t)=1$ and $f(x)=x$, we refer to the articles by Ladas and Sficas [20], Grammatikopoulos et al. [10] and Zhang [33] and the references cited therein. For $P(t)$ equal to a constant, $a(t)=1$ and $f(x)=x$ we refer to the articles by Grammatikopoulos, Grove and Ladas [12] Zhang [33] and Saker and Elabbasy [24]. Grammatikopouolos et al. [11] considered the neutral delay differential equation (1.1) when $a(t)=1$ and $f(x)=x$ and presented some finite sufficient conditions for 
oscillation of all solutions when $P(t)$ takes some values in the interval $[0,1]$. Chuanxi and Ladas [7] and Kubiaczyk and Saker [19] considered the neutral delay differential equation (1.1) when $a(t)=1$ and $f(x)=x$ and established new sufficient conditions for oscillation of all solutions under less restrictive hypotheses on $P(t)$. All the above mentioned papers except [24] given the oscillation conditions for equation (1.1) when $P(t) \leq 1$ under the condition

$$
\int_{t_{0}}^{\infty} Q(s) d s=\infty
$$

In the case when (1.5) does not hold (in this case equation (1.1) is called has integrally small coefficients) Yu, Wang and Chuanxi [28] considered equation (1.1) when $P(t) \equiv 1$ and relaxed the condition (1.5) to the condition

$$
\int_{t_{0}}^{\infty} s Q(s) \int_{s}^{\infty} Q(u) d u d s=\infty .
$$

In fact, Chen, Yu and Huang [6] observed that for the equation (1.1), it is sufficient to have a point $t^{*} \geq t_{0}$ so that

$$
P\left(t^{*}+k \tau\right) \leq 1 \quad k=0,1,2, \ldots
$$

without the assumptions (1.5) and (1.6) and proved a comparison theorem for oscillation of equation (1.1) with the absence of positive solutions of the corresponding delay differential inequality. They succeeded in getting oscillation theorem which involve joint behavior of $P$ and $Q$ (for example see [6, Theorems 3 and 5]) and using the condition

$$
P(t-\sigma) Q(t) \leq Q(t-\tau)
$$

to transfer the equation (1.1) to the inequality

$$
\frac{d}{d t}[y(t)-y(t-\tau)]+Q(t) y(t-\sigma) \leq 0, t \geq t_{0},
$$

and using the results in $\mathrm{Yu}$, Wang and Chuanxi [28] with the condition (1.6) in the proofs of the main results, and obtained some finite sufficient conditions for oscillation of all solutions. However, most of the results in the literature involve conditions placed separately on $\mathrm{Q}$ mimicking the conditions on $Q$ sufficient for oscillation of (1.1) and placing conditions on $P$ which allow extension of arguments used in the case where $P(t) \equiv 1$ as in $\mathrm{Yu}$, Wang and Chuanxi [28]. Recently Li and Saker [22] considered the equation (1.1) when $a(t)=1$ and given some finite sufficient conditions for oscillation of all solutions and applied these results to the logistic neutral delay differential equations. For further oscillation results when (1.5) does not hold we refer the reader to the articles by $\mathrm{Yu}[26,27]$ and $\mathrm{Yu}$ et al. [29, $30,31]$.

Our aim in this paper in Section 2 is to give some new integral sufficient conditions for oscillation of all solutions of equation (1.1) and show that 
the combined growth of $P$ and $Q$ without the condition (1.8) in the linear case can give oscillation even when (1.5) and (1.6) fail. Our results extend and improve some well known results for the oscillation of (1.1), and improve some theorems about oscillation of the linear neutral delay differential equations. In Section 3 some examples are considered to illustrate our main results and in Section 4 we apply our results to the neutral logistic equation with variable coefficients to give some oscillation criteria for all positive solutions about its positive steady state.

In the sequel, when we write a functional inequality we will assume that it holds for all sufficient large values of $t$.

Before stating our main results we need the following lemma.

Lemma 1.1. Assume that (1.2), (1.3) hold, and there exist $t^{*} \geq t_{0}$ such that

$$
\frac{P\left(t^{*}+i \tau\right)}{a\left(t^{*}+(i-1) \tau\right)} \leq 1 \quad \text { for } i=0,1,2, \ldots
$$

Let $x(t)$ be an eventually positive solution of equation (1.1), and set

$$
y(t)=a(t) x(t)-P(t) x(t-\tau) .
$$

Then we have eventually

$$
y(t)>0 .
$$

Proof. Let $t_{1} \geq t_{0}$ be such that $x(t)>0, x(t-\sigma)>0$ and $x(t-\tau)>0$ for $t \geq t_{1}$. Then by (1.1) and (1.11) we have

$$
y^{\prime}(t)=-Q(t) f(x(t-\sigma))<0 \quad \text { for } t \geq t_{1}
$$

which implies that $y(t)$ is nonincreasing on $\left[t_{1}, \infty\right)$ and does not equal a constant eventually. Hence if (1.12) does not hold, then eventually

$$
y(t)<0, \quad t \geq t_{1} .
$$

Therefore there exist $t_{2} \geq t_{1}$ and $M>0$ such that

$$
y(t)<-M, \quad t \geq t_{2} .
$$

Set $z(t)=a(t) x(t)>0$, then

$$
z(t)<-M+\frac{P(t)}{a(t-\tau)} z(t-\tau), \quad t \geq t_{2} .
$$

Now we chose a positive integer i to be such that $t^{*}+i \tau>t_{2}$. Then by (1.10) and (1.13) we get

$$
z\left(t^{*}+i \tau\right) \leq-M(i+1)+z\left(t^{*}\right) \rightarrow-\infty \text { as } i \rightarrow \infty
$$

which contradicts the fact that $z(t)$ is eventually positive. Then (1.12) holds. The proof is complete. 


\section{Main results}

In this section we will establish some sufficient conditions for oscillation of all solutions of equation (1.1).

Theorem 2.1. Assume that (1.2), (1.3) and (1.10) hold,

$$
0<d \leq \liminf _{t \rightarrow \infty} \int_{t}^{t+\sigma} \frac{Q_{1}(s)}{a(s-\sigma)} d s \quad \text { for } t \geq t_{0},
$$

and

$$
\int_{t_{0}}^{\infty} \frac{Q_{1}(t)}{a(t-\sigma)} \exp \left[\int_{t}^{t+\sigma} \frac{Q_{1}(s)}{a(s-\sigma)} d s\right] d t=\infty
$$

where $Q_{1}(t)=Q(t) /(\beta+\varepsilon)$ for some small positive constant $\varepsilon$. Then every solution of equation (1.1) oscillates.

Proof. Assume, by the way of contradiction, that equation (1.1) has an eventually nonoscillatory solution. Without loss of generality we assume that equation (1.1) has an eventually positive solution $x(t)$ (the case that $x(t)$ is negative is similar and will be omitted). Set $x(t)>0$ and $x(t-\sigma)>0$, for $t \geq t_{0}$. From (1.3) since the limit exists, we can assume that there exists $T_{\varepsilon}$ sufficiently large such that for $t \geq T_{\varepsilon}, 0<x(t-\sigma)$ and $x(t-\sigma) /(\beta+\varepsilon) \leq$ $f(x(t-\sigma)) \leq x(t-\sigma) /(\beta-\varepsilon)$. Set $y(t)$ as in (1.11), then from equation (1.1) and Lemma 1.1, $y(t)$ is a positive function and satisfies the inequalities:

$$
y^{\prime}(t)+Q(t) \frac{x(t-\sigma)}{\beta+\varepsilon} \leq 0, y^{\prime}(t)+Q(t) \frac{x(t-\sigma)}{\beta-\varepsilon} \geq 0, t \geq T_{\varepsilon} .
$$

Then from (1.11) and (2.3) we have

$$
\begin{aligned}
y^{\prime}(t) & \leq-\frac{Q_{1}(t)}{a(t-\sigma)} y(t-\sigma)-\frac{Q_{1}(t) P(t-\sigma)}{a(t-\sigma)} x(t-\tau-\sigma) \\
& \leq-\frac{Q_{1}(t)}{a(t-\sigma)} y(t-\sigma)+\frac{Q_{1}(t)}{Q_{1}(t-\tau)} \frac{P(t-\sigma)}{a(t-\sigma)} y^{\prime}(t-\tau), t \geq T_{\varepsilon} .
\end{aligned}
$$

Hence $y(t)$ is positive and satisfies the inequality

$$
y^{\prime}(t)-\frac{Q_{1}(t)}{Q_{1}(t-\tau)} \frac{P(t-\sigma)}{a(t-\sigma)} y^{\prime}(t-\tau)+\frac{Q_{1}(t)}{a(t-\sigma)} y(t-\sigma) \leq 0 .
$$

Set

$$
\lambda(t)=-\frac{y^{\prime}(t)}{y(t)}
$$


Then (2.4) reduces to

$$
\begin{aligned}
\lambda(t) \geq & \lambda(t-\tau) \frac{Q_{1}(t)}{a(t-\sigma)} \frac{P(t-\sigma)}{Q_{1}(t-\tau)} \exp \left(\int_{t-\tau}^{t} \lambda(s) d s\right) \\
& +\frac{Q_{1}(t)}{a(t-\sigma)} \exp \left(\int_{t-\sigma}^{t} \lambda(s) d s\right) .
\end{aligned}
$$

It is obvious that $\lambda(t)>0$ for $t \geq t_{0}$. From (2.6) it is clear that

$$
\lambda(t) \geq \bar{Q}(t) \exp \left(\int_{t-\sigma}^{t} \lambda(s) d s\right)
$$

with $\bar{Q}(t)=Q_{1}(t) /[a(t-\sigma)]$. Let $A(t)=\exp \left(\int_{t}^{t+\sigma} \bar{Q}(s) d s\right)$, then

$$
\lambda(t) \geq \bar{Q}(t) \exp \left[\frac{1}{A(t)} A(t) \int_{t-\sigma}^{t} \lambda(s) d s\right] .
$$

By using the inequality

$$
e^{x / r} \geq 1+\frac{x}{r^{2}} \quad \text { for } x \geq 0, r \geq 1
$$

we have from the inequality (2.7) that

$$
A(t) \lambda(t)-\bar{Q}(t) \int_{t-\sigma}^{t} \lambda(s) d s \geq \bar{Q}(t) A(t) .
$$

Then for $N>T$,

$$
\int_{T}^{N} \lambda(t) A(t) d t-\int_{T}^{N} \bar{Q}(t) \int_{t-\sigma}^{t} \lambda(s) d s d t \geq \int_{T}^{N} \bar{Q}(t) A(t) d t .
$$

By interchanging the order of integration, we find that

$$
\int_{T}^{N} \bar{Q}(t)\left(\int_{t-\sigma}^{t} \lambda(s) d s\right) d t \geq \int_{T}^{N-\sigma} \lambda(t)\left(\int_{t}^{t+\sigma} \bar{Q}(s) d s\right) d t
$$

Hence

$$
\begin{aligned}
& \int_{T}^{N} \lambda(t) A(t) d t-\int_{T}^{N-\sigma} \lambda(t)\left(\int_{t}^{t+\sigma} \bar{Q}(s) d s\right) d t \\
& \geq \int_{T}^{N} \lambda(t) A(t) d t-\int_{T}^{N} \bar{Q}(t) \int_{t-\sigma}^{t} \lambda(s) d s d t .
\end{aligned}
$$

From (2.9) and (2.10), it follows that

$$
\int_{T}^{N} \lambda(t) A(t) d t+\int_{N-\sigma}^{T} \lambda(t)\left(\int_{t}^{t+\sigma} \bar{Q}(s) d s\right) d t \geq \int_{T}^{N} \bar{Q}(t) A(t) d t .
$$

On the other hand from the definition of $\lambda(t)$, then from (2.6) $y(t)$ is positive function and satisfies the delay differential inequality

$$
y^{\prime}(t)+\bar{Q}(t) y(t-\sigma) \leq 0
$$


Integrating the last inequality from $t$ to $t+\sigma$ we have

$$
y(t+\sigma)-y(t)+\int_{t}^{t+\sigma} \bar{Q}(s) y(s-\sigma) d s \leq 0 .
$$

Then

$$
y(t)>\int_{t}^{t+\sigma} \bar{Q}(s) y\left(s-\sigma_{1}\right) d s>y(t) \int_{t}^{t+\sigma} \bar{Q}(s) d s
$$

which implies that

$$
d \leq \int_{t}^{t+\sigma} \bar{Q}(s) d s<1 \text { and } e^{d} \leq A(t)<e .
$$

Therefore

$$
A(t)>\int_{t}^{t+\sigma} \bar{Q}(s) d s
$$

Then

$$
\int_{T}^{N} \lambda(t) A(t) d t+\int_{N-\sigma}^{T} \lambda(t) A(t) d t \geq \int_{T}^{N} \bar{Q}(t) A(t) d t .
$$

Then from (2.12) and (2.13) we have

$$
\int_{N-\sigma}^{N} \lambda(t) d t \geq \frac{1}{e} \int_{T}^{N} \bar{Q}(t) A(t) d t
$$

or

$$
\log \frac{y(N-\sigma)}{y(N)} \geq \frac{1}{e} \int_{T}^{N} \bar{Q}(t) A(t) d t .
$$

In view of $(2.2)$ we have

$$
\lim _{t \rightarrow \infty} \frac{y(t-\sigma)}{y(t)}=\infty .
$$

Because of $d \leq \liminf _{t \rightarrow \infty} \int_{t}^{t+\sigma} \bar{Q}(s) d s$ there exists a sequence $\left\{t_{k}\right\}, t_{k} \rightarrow \infty$ as $k \rightarrow \infty$ and there exist $\zeta_{k} \in\left(t_{k}, t_{k}+\sigma\right)$ for every $k$ such that

$$
\int_{t_{k}}^{\zeta_{k}} \bar{Q}(s) d s \geq \frac{d}{2} \quad \text { and } \quad \int_{\zeta_{k}}^{t_{k}+\sigma} \bar{Q}(s) d s \geq \frac{d}{2} .
$$

Integrating both sides of the inequality $(2.11)$ over the intervals $\left[t_{k}, \zeta_{k}\right]$ and $\left[\zeta_{k}, t_{k}+\sigma\right]$, we have

$$
y\left(\zeta_{k}\right)-y\left(t_{k}\right)+\int_{t_{k}}^{\zeta_{k}} \bar{Q}(s) y(s-\sigma) d s \leq 0
$$

and

$$
y\left(t_{k}+\sigma\right)-y\left(\zeta_{k}\right)+\int_{\zeta_{k}}^{t_{k}+\sigma} \bar{Q}(s) y(s-\sigma) d s \leq 0 .
$$


From (2.16), (2.17) and (2.18), we have

$$
-y\left(t_{k}\right)+\frac{d}{2} y\left(\zeta_{k}-\sigma\right) \leq 0 \quad \text { and } \quad-y\left(\zeta_{k}\right)+\frac{d}{2} y\left(t_{k}\right) \leq 0 .
$$

Then

$$
\frac{y\left(\zeta_{k}-\sigma\right)}{y\left(\zeta_{k}\right)} \leq\left(\frac{2}{d}\right)^{2}
$$

which contradicts (2.15). Therefore, every solution of equation (1.1) oscillates.

The following theorems are improved Theorem 2.1 which indicate that the oscillation conditions of all solutions of equation (1.1) depend on $P$ and $Q$.

Theorem 2.2. Assume that (1.2), (1.3) and (1.10) hold,

$$
0<d \leq \liminf _{t \rightarrow \infty} \int_{t}^{t+\sigma} \bar{Q}_{1}(s) d s \text { for } t \geq t_{0}
$$

and

$$
\int_{t_{0}}^{\infty} \bar{Q}_{1}(t) \exp \left(\int_{t}^{t+\sigma} \bar{Q}_{1}(s) d s\right) d t=\infty .
$$

Then every solution of equation (1.1) oscillates, where

$$
\bar{Q}_{1}(t)=\frac{Q_{1}(t) P(t-\sigma)}{a(t-\sigma) a(t-\tau-\sigma)}
$$

Proof. Without loss of generality, we assume that equation (1.1) has an eventually positive solution $x(t)$. Then from Theorem 2.1 then $y(t)>0$ and its generalized equation is given by (2.6). From (2.6) one can see that $\lambda(t) \geq$ $Q_{1}(t) /[a(t-\sigma)]$, then $\lambda(t-\tau) \geq Q_{1}(t-\tau) /[a(t-\tau-\sigma)]$, substituting in (2.6) we have

$$
\begin{aligned}
\lambda(t) \geq & \left.\frac{Q_{1}(t) P(t-\sigma)}{a(t-\sigma) a(t-\tau-\sigma)} \exp \left(\int_{t-\tau}^{t} \lambda(s) d s\right)\right) \\
& +\frac{Q_{1}(t)}{a(t-\sigma)} \exp \left(\int_{t-\sigma}^{t} \lambda(s) d s\right) .
\end{aligned}
$$

It is obvious that $\lambda(t)>0$ for $t \geq t_{0}$ and then

$$
\lambda(t) \geq \frac{Q_{1}(t) P(t-\sigma)}{a(t-\sigma) a(t-\tau-\sigma)} \exp \left(\int_{t-\tau}^{t} \lambda(s) d s\right) .
$$

The remainder of the proof is similar to the proof of Theorem 2.1 and will be omitted. 
Theorem 2.3. Assume that (1.2), (1.3) and (1.10) hold, $\tau \geq \sigma$,

$$
\begin{gathered}
d \leq \liminf _{t \rightarrow \infty} \int_{t}^{t+\sigma} \bar{Q}_{2}(s) d s \\
\int_{t_{0}}^{\infty} \bar{Q}_{2}(t) \exp \left(\int_{t}^{t+\sigma} \bar{Q}_{2}(s) d s\right) d t=\infty,
\end{gathered}
$$

then every solution of equation (1.1) oscillates, where

$$
\bar{Q}_{2}(t)=\frac{1}{a(t-\sigma)}\left[\frac{Q_{1}(t) P(t-\sigma)}{a(t-\tau-\sigma)}+Q_{1}(t)\right] .
$$

Proof. The proof is similar to the proof of Theorem 2.1 from the inequality (2.6) and will be omitted.

Theorem 2.4. Assume that (1.2), (1.3) and (1.10) hold and $\tau \geq \sigma$,

$$
\begin{gathered}
d \leq \liminf _{t \rightarrow \infty} \int_{t}^{t+\sigma} \bar{Q}_{3}(s) d s \\
\int_{t_{0}}^{\infty} \bar{Q}_{3}(t) \exp \left(\int_{t}^{t+\sigma} \bar{Q}_{3}(s) d s\right) d t=\infty .
\end{gathered}
$$

then every solution of equation (1.1) oscillates, where

$$
\bar{Q}_{3}(t)=\frac{Q_{1}(t) P(t-\sigma) P(t-\tau-\sigma)}{a(t-\tau-\sigma) a(t-2 \tau-\sigma)}+\frac{Q_{1}(t)}{a(t-\sigma)} .
$$

Proof. Without loss of generality, we assume that equation (1.1) has an eventually positive solution $x(t)$. As in Theorem 2.1 from equation (2.6) it is obvious that $\lambda(t)>0$ for $t \geq t_{0}$, and $\lambda(t) \geq Q_{1}(t) /[a(t-\sigma)]$. Hence $\lambda(t-\tau) \geq Q_{1}(t-\tau) /[a(t-\tau-\sigma)]$ and

$$
\begin{aligned}
\lambda(t) \geq & \frac{Q_{1}(t) P(t-\sigma)}{a(t-\sigma) a(t-\tau-\sigma)} \exp \left(\int_{t-\tau}^{t} \lambda(s) d s\right) \\
& +\frac{Q_{1}(t)}{a(t-\sigma)} \exp \left(\int_{t-\sigma}^{t} \lambda(s) d s\right)
\end{aligned}
$$

which guarantees that

$$
\lambda(t) \geq \frac{Q_{1}(t) P(t-\sigma)}{a(t-\sigma) a(t-\tau-\sigma)}
$$

and then

$$
\lambda(t-\tau) \geq \frac{Q_{1}(t-\tau) P(t-\tau-\sigma)}{a(t-\tau-\sigma) a(t-2 \tau-\sigma)} .
$$


From (2.6) we have

$$
\begin{aligned}
\lambda(t) \geq & \frac{Q_{1}(t) P(t-\sigma) P(t-\tau-\sigma)}{a(t-\tau-\sigma) a(t-2 \tau-\sigma)} \exp \left(\int_{t-\tau}^{t} \lambda(s) d s\right) \\
& +\frac{Q_{1}(t)}{a(t-\sigma)} \exp \left(\int_{t-\sigma}^{t} \lambda(s) d s\right)
\end{aligned}
$$

as $\tau \geq \sigma$ we obtain

$$
\begin{aligned}
\lambda(t) \geq & \left(\frac{Q_{1}(t) P(t-\sigma) P(t-\tau-\sigma)}{a(t-\tau-\sigma) a(t-2 \tau-\sigma)}\right. \\
& \left.+\frac{Q_{1}(t)}{a(t-\sigma)}\right) \exp \left(\int_{t-\sigma}^{t} \lambda(s) d s\right) .
\end{aligned}
$$

The remainder of the proof is similar to the proof of Theorem 2.1 and will be omitted.

Theorem 2.5. Assume that (1.2), (1.3) and (1.10) hold,

$$
\frac{1}{e}<\int_{t}^{t+\sigma_{1}} \bar{Q}(s) d s
$$

and

$$
\int_{t_{0}}^{\infty} \bar{Q}(t) \log \left(e \int_{t}^{t+\sigma_{1}} \bar{Q}(s) d s\right) d t=\infty .
$$

Then every solution of equation (1.1) oscillates.

Proof. The proof is similar to the proof of Theorem 2.1 by choosing

$$
A(t)=\log \left\{e \int_{t}^{t+\sigma_{1}} \bar{Q}(s) d s\right\}
$$

and will be omitted.

Theorem 2.6. Assume that (1.2), (1.3) and (1.10) hold,

$$
\frac{1}{e} \leq \int_{t}^{t+\sigma_{1}} \bar{Q}(s) d s
$$

and

$$
\int_{t_{0}}^{\infty} \bar{Q}(t)\left\{\exp \left(\int_{t}^{t+\sigma_{1}} \bar{Q}(s) d s-\frac{1}{e}\right) d s-1\right\} d t=\infty .
$$

Then every solution of equation (1.1) oscillates. 
Proof. The proof is similar to the proof of Theorem 2.1 by choosing

$$
A(t)=\left\{\exp \left(\int_{t}^{t+\sigma_{1}} \bar{Q}(s) d s-\frac{1}{e}\right) d s-1\right\}
$$

and will be omitted.

In fact, if we take

$$
\begin{aligned}
& \bar{Q}_{1}(t)=\frac{Q_{1}(t) P(t-\sigma)}{a(t-\sigma) a(t-\tau-\sigma)}, \\
& \bar{Q}_{2}(t)=\frac{1}{a(t-\sigma)}\left[\frac{Q_{1}(t) P(t-\sigma)}{a(t-\tau-\sigma)}+Q_{1}(t)\right], \\
& \bar{Q}_{3}(t)=\frac{Q_{1}(t) P(t-\sigma) P(t-\tau-\sigma)}{a(t-\tau-\sigma) a(t-2 \tau-\sigma)}+\frac{Q_{1}(t)}{a(t-\sigma)},
\end{aligned}
$$

then respectively we have the following new sufficient conditions for oscillation of all solutions of equation (1.1).

Theorem 2.7. Assume that (1.2), (1.3) and (1.10) hold,

$$
\frac{1}{e}<\int_{t}^{t+\tau} \overline{Q_{1}}(s) d s
$$

and

$$
\int_{t_{0}}^{\infty} \overline{Q_{1}}(t) \log \left(e \int_{t}^{t+\tau} \overline{Q_{1}}(s) d s\right) d t=\infty .
$$

Then every solution of equation (1.1) oscillates.

Theorem 2.8. Assume that (1.2), (1.3) and (1.10) hold,

and

$$
\frac{1}{e} \leq \int_{t}^{t+\sigma} \overline{Q_{1}}(s) d s
$$

$$
\int_{t_{0}}^{\infty} \overline{Q_{1}}(t)\left\{\exp \left(\int_{t}^{t+\sigma} \bar{Q}_{1}(s) d s-\frac{1}{e}\right) d s-1\right\} d t=\infty .
$$

Then every solution of equation (1.1) oscillates.

Theorem 2.9. Assume that (1.2), (1.3) and (1.10) hold,

$$
\frac{1}{e}<\int_{t}^{t+\sigma} \overline{Q_{2}}(s) d s
$$

and

$$
\int_{t_{0}}^{\infty} \overline{Q_{2}}(t) \log \left(e \int_{t}^{t+\sigma} \overline{Q_{2}}(s) d s\right) d t=\infty .
$$

Then every solution of equation (1.1) oscillates. 
Theorem 2.10. Assume that (1.2), (1.3) and (1.10) hold,

$$
\frac{1}{e} \leq \int_{t}^{t+\sigma} \overline{Q_{2}}(s) d s
$$

and

$$
\int_{t_{0}}^{\infty} \overline{Q_{2}}(t)\left\{\exp \left(\int_{t}^{t+\sigma} \overline{Q_{2}}(s) d s-\frac{1}{e}\right) d s-1\right\} d t=\infty .
$$

Then every solution of equation (1.1) oscillates.

Theorem 2.11. Assume that (1.2), (1.3) and (1.10) hold,

$$
\frac{1}{e}<\int_{t}^{t+\sigma} \overline{Q_{3}}(s) d s
$$

and

$$
\int_{t_{0}}^{\infty} \overline{Q_{3}}(t) \log \left(e \int_{t}^{t+\sigma} \overline{Q_{3}}(s) d s\right) d t=\infty
$$

Then every solution of equation (1.1) oscillates.

Theorem 2.12. Assume that (1.2), (1.3) and (1.10) hold,

$$
\frac{1}{e} \leq \int_{t}^{t+\sigma} \overline{Q_{3}}(s) d s
$$

and

$$
\int_{t_{0}}^{\infty} \overline{Q_{3}}(t)\left\{\exp \left(\int_{t}^{t+\sigma} \overline{Q_{3}}(s) d s-\frac{1}{e}\right) d s-1\right\} d t=\infty .
$$

Then every solution of equation (1.1) oscillates.

Remark 2.1. Our results can be extended to the more the general equation

$$
\frac{d^{n}}{d t^{n}}\left[a(t) x(t)-\sum_{j=1}^{m} P_{j}(t) x\left(t-\tau_{j}\right)\right]+\sum_{i=1}^{n} Q_{i}(t) f\left(x\left(t-\sigma_{i}\right)\right)=0, t \geq t_{0} .
$$

Due to limited space, their statements are omitted here. 


\section{Examples}

In this section we introduce some examples to illustrate our results.

Example 3.1. Consider the neutral delay differential equation

$$
\left[x(t)-\left(\frac{3}{2}+\sin t\right) x(t-\pi)\right]^{\prime}+\frac{3}{2}\left(\left(\sqrt{2}+\frac{1}{e}\right) \frac{2}{\pi}+\cos t\right)\left(e^{x(t-\pi / 2)}-1\right)=0
$$

Here $\sigma=\pi / 2$ and

$$
f(u)=e^{u}-1
$$

with $\beta=1$ and $\varepsilon=1 / 2$, then

$$
Q_{1}(t)=\left(\sqrt{2}+\frac{1}{e}\right) \frac{2}{\pi}+\cos t>0 \quad \text { for } t \geq 0
$$

and

$$
\int_{t-\pi / 2}^{t} Q_{1}(s) d s=\int_{t-\pi / 2}^{t}\left(\left(\sqrt{2}+\frac{1}{e}\right) \frac{2}{\pi}+\cos s\right) d s=\sqrt{2}+\frac{1}{e}+\sin t+\cos t .
$$

Hence

$$
\liminf _{t \rightarrow \infty} \int_{t-\pi / 2}^{t} Q_{1}(s) d s=\frac{1}{e} .
$$

Then according to Theorem 3.3 in [22] equation (3.1) cannot has an oscillatory solution, but one can prove by Theorem 2.1 that every solution of equation (3.1) oscillates.

Example 3.2. Consider the neutral delay differential equation

$$
\begin{array}{r}
{\left[x(t)-\left(\frac{3}{2}+\sin t\right) x(t-\pi)\right]^{\prime}+\frac{3}{2} \frac{0.6}{\alpha \pi+\sqrt{2}}(2 \alpha+\cos t)\left(e^{x(t-\pi / 2)}-1\right)=0} \\
t \geq 0
\end{array}
$$

with $\sigma=\pi / 2$,

$$
f(u)=e^{u}-1
$$

with $\beta=1, \varepsilon=1 / 2, a(t)=1$, and $\alpha=\sqrt{2}(0.6 e+1) /[\pi(0.6 e-1)]$

$$
Q_{1}(t)=\frac{0.6}{\alpha \pi+\sqrt{2}}(2 \alpha+\cos t)>0 \quad \text { for } t \geq 0
$$

and

$$
\int_{t-\pi / 2}^{t} Q_{1}(s) d s=\int_{t-\pi / 2}^{t} \frac{0.6}{\alpha \pi+\sqrt{2}}(2 \alpha+\cos s) d s .
$$

Hence

$$
\liminf _{t \rightarrow \infty} \int_{t-\pi / 2}^{t} Q_{1}(s) d s=\frac{1}{e}
$$


and

$$
\limsup _{t \rightarrow \infty} \int_{t-\pi / 2}^{t} Q_{1}(s) d s=0.6 .
$$

Then Theorem 3.3 in [22] is failed to apply on the equation (3.2), but one can see by Theorem 2.1 that every solution of equation (3.2) oscillates.

Example 3.3. Consider the neutral delay differential equation

$$
\begin{aligned}
{\left[x(t)-\left(\frac{3}{2}+\sin t\right) x(t-\pi)\right]^{\prime}+\frac{3}{2}\left(\frac{1}{e}+\frac{1}{t+1}\right)\left(1-e^{-x(t-1)}\right) } & =0, \\
t & \geq 0
\end{aligned}
$$

with $\sigma=1$,

$$
f(u)=1-e^{-u}
$$

with $\beta=1, \varepsilon=1 / 2$, and $a(t)=1$,

$$
Q_{1}(t)=\left(\frac{1}{e}+\frac{1}{t+1}\right)
$$

for $t \geq 0$ and

$$
\int_{t-1}^{t} Q_{1}(s) d s=\int_{t-1}^{t}\left(\frac{1}{e}+\frac{1}{s+1}\right) d s=\log \frac{t+1}{t}+\frac{1}{e}
$$

Hence

$$
\liminf _{t \rightarrow \infty} \int_{t-1}^{t} Q_{1}(s) d s=\frac{1}{e}
$$

For $T>1$ we have

$$
\begin{aligned}
& \int_{1}^{T} Q_{1}(t) \exp \left(\int_{t}^{t+1} Q_{1}(s) d s\right) d t=\int_{1}^{T}\left(\frac{1}{e}+\frac{1}{t+1}\right) \exp \left(\log \frac{t+2}{t+1}+1\right) d t \\
& \geq e \int_{1}^{T}\left(\frac{1}{e}+\frac{1}{t+1}\right)\left(\log \frac{t+2}{t+1}+1\right) d t \rightarrow \infty
\end{aligned}
$$

as $T \rightarrow \infty$ where $e^{x} \geq e x$ for all real $x$. Then by Theorem 2.1 every solution of equation (3.3) oscillates.

\section{Oscillation in non-autonomous neutral delay logistic equation}

The scalar autonomous ordinary differential equation

$$
N^{\prime}(t)=r N(t)\left[1-\frac{N(t)}{K}\right]
$$

is known as the logistic equation in mathematical ecology and it is a prototype in the modelling in the dynamics of single-species population systems whose biomass or density is denoted by a differentiable function $N(t)$. The 
constant $r$, is called the growth rate and $K$, is called the carrying capacity of the habitat. Hutchinson [17] suggested the following modification

$$
N^{\prime}(t)=r N(t)\left[1-\frac{N(t-\tau)}{K}\right] \text {. }
$$

Equation (4.1) is commonly known as the "delay equation" and has been extensively investigated by numerous authors (see for example Wright [25], Kakutani and Markus [18]). Gyori [14] considered the neutral delay logistic equation with constant coefficients of the form,

$$
N^{\prime}(t)=N(t)\left[r\left(1-\frac{N(t-\sigma)}{K}\right)+c N^{\prime}(t-\tau)\right]
$$

and established oscillation criteria for all positive solutions.

The effects of varying environment are often important in dynamical nature of populations, then we consider the non-autonomous neutral delay equation

$$
N^{\prime}(t)=N(t)\left[r(t)\left(1-\frac{N(t-\sigma)}{K}\right)+c N^{\prime}(t-\tau)\right]
$$

where

$$
r \in C\left[\left[t_{0}, \infty\right), \mathbb{R}^{+}\right], \quad K, \tau, \sigma, c \in(0, \infty)
$$

and $r(t)$ is the growth rate function, $K$ is the carrying capacity of the environment and $c$ is the growth rate associated with the growth rate at time $t-\tau$.

With equation (4.3) one associate an initial condition of the form,

$$
N(t)=\phi(t) \quad \text { for }-\gamma \leq t \leq 0, \phi \in C\left[[-\tau, 0], \mathbb{R}^{+}\right] \text {and } \phi(0)>0
$$

where $\gamma=\max \{\tau, \sigma\}$, then by the method of steps, the initial value problem (4.3) and (4.5) has a unique solution $N(t)$ which is valid for $t \geq 0$. We will only consider those solutions $N(t)$ which are positive. Note that such solution exist because if $\phi(0)>0$, then $N(t)>0$ for $t \geq 0$. In [22] Li and Saker presented some finite sufficient conditions for oscillation of all positive solutions of equation (4.3) about $K$ when $0<c(t)<1$. In this section we introduce some new infinite sufficient conditions for oscillation of all positive solution of equation (4.3) when $c(t)=c$ is a constant by applying one of the above theorems.

Theorem 4.1. Assume that (4.4) holds, $0<c<1$,

$$
0<\liminf _{t \rightarrow \infty} \int_{t}^{t+\sigma} r(s) d s
$$


and

$$
\int_{t_{0}}^{\infty} r(t) \log \left[e \int_{t}^{t+\sigma} r(s) d s\right] d t=\infty .
$$

Then every solution of equation (4.3) oscillates.

Proof. The change of variables

$$
N(t)=K e^{x(t)}
$$

reduces equation (4.3) to the delay equation

$$
\frac{d}{d t}[x(t)-c x(t-\tau)]+r(t)\left[e^{x(t-\sigma)}-1\right]=0
$$

Clearly, $N(t)$ oscillates about $K$, if and only if $x(t)$ oscillates about zero. From (4.7) we have

$$
\frac{d}{d t}[x(t)-c x(t-\tau)]+r(t) f(x(t-\sigma))=0
$$

with

$$
f(u)=e^{u}-1 .
$$

It is clear that

$$
f \in C[\mathbb{R}, \mathbb{R}], \quad u f(u)>0 \text { for } u \neq 0, \quad \lim _{u \rightarrow 0} \frac{u}{f(u)}=1 .
$$

Then by Theorem 2.1 and the condition (4.6) every solution of equation (4.7) oscillates. Then every positive solution of equation (4.3) oscillates about $K$.

One can use equation (4.7) and the above theorem to give several new sufficient conditions for oscillation of all positive solutions of equation (4.3) about $K$. The details are left to the reader.

Acknowledgement The authors thanks the referee for his helpful suggestions and his excellent list of the references [26, 27, 29, 30, 31]. 


\section{References}

[1] Agarwal, R. P., Grace, S, R., O'Regan, D., Oscillation Theory for Difference and Functional Differential Equations, Kluwer, Dordrecht, 2000.

[2] Agarwal, R. P., Grace, S. R., O'Regan, D., Oscillation Theory for Second Order Dynamic Equations, (to appear).

[3] Bainov, D. D., Mishev, D. P., Oscillation Theory for Neutral Differential Equations with Delay, Adam Hilger, New York, 1991.

[4] Boe, E., Chang, V., Dynamics of delayed systems under feedback control, Chem. Engrg. Sci. 44 (1989), 1281-1294.

[5] Brayton, R. K., Willoughby, R. A., On the numerical integration of a symmetric system of difference-differential equations of neutral type, J. Math. Anal. Appl., 18 (1976), 182-189.

[6] Chen, M. P, Yu, J. S., Huang, L. H., Oscillations of first order neutral differential equations with variable coefficients, J. Math. Anal. Appl. 185 (1994), 288-301.

[7] Chuanxi Q., Ladas, G., Oscillation of first-order neutral equations with variable coefficients, Monatsh. Math. 109 (1990), 103-111.

[8] Driver, D. R., A mixed neutral systems, Nonlinear Anal. 8 (1984), 155-158.

[9] Erbe, L. H., Kong, Q., Zhang, B. G., Oscillation Theory for Functional Differential Equations, Dekker, New York, 1995.

[10] Grammatikopoulos, M. K., Sficas, Y. G., Ladas, G., Oscillation and asymptotic behavior of neutral differential equations with deviating argument, Appl. Anal. 22 (1986), 1-19.

[11] Grammatikopoulos, M. K., Sficas, Y. G., Ladas, G., Oscillation and asymptotic behavior of neutral equations with variable coefficients, Rad. Mat. 2 (1986), 279-303.

[12] Grammatikopoulos, M. K., Grove, E. A., Ladas, G., Oscillation of first-order neutral delay differential equations, J. Math. Anal. Appl. 120 (1986), 510-520.

[13] Guo, B. C., Oscillation of neutral functional differential equations (in Chinese), Acta Math. Appl. Sinica 19 (1996), 513-522.

[14] Gyori, I., Oscillation and comparison results in neutral differential equations and their applications to the delay logistic equation, Comp. Math. Appl. 18 (1989), 883-906.

[15] Gyori, I., Ladas, G., Oscillation Theory of Delay Differential Equations with Applications, Clarendon Press, Oxford, 1991.

[16] Hale, J. K., Theory of Functional Differential Equations, Springer-Verlag, New York, 1977.

[17] Hutchinson, G. E., Circular casual systems in ecology, Ann. New York Acad. Sci. 50 (1948), 221-240.

[18] Kakutani, S, Markus, L., On the nonlinear difference-differential equation $\dot{y}(t)=$ $[A-B y(t-\tau)] y(t)$, Contrib. Theory Nonlinear Oscill. 4 (1958), Princeton University Press., Princeton, NJ, 1-18.

[19] Kubiaczyk, I, Saker, S. H., Oscillation of solution of neutral delay differential equations, Math. Slovaca 52 (2002), 343-359.

[20] Ladas, G., Sficas, Y. G., Oscillation of neutral delay differential equations, Canad. Math. Bull. 29(4) (1986), 438-445.

[21] Ladde, G. S., Lakshmikantham, V., Zhang, B. Z., Oscillation Theory of Differential Equations with Deviating Arguments, Marcel Dekker, New York, 1987.

[22] Li, W. T., Saker, S. H., Oscillation of nonlinear neutral delay differential equations and apllications, Ann. Polon. Math. 77 (2001), 39-51.

[23] Popov, E. P., Automatic Regulation and Control (in Russian), Nauka, Moscow, 1966. 
[24] Saker, S. H., Elabbasy, E. M., Oscillation of first order neutral delay differential equations, Kyungpook Math. J. 41(2) (2001), 311-321.

[25] Wright, E. M., A nonlinear difference-differential equations, J. Reine Angew. Math. 194 (1955), 66-87.

[26] Yu, J. S., Oscillation of neutral differential equations with oscillating coefficients, Czechoslovak Math. J. 44 (1994), 611-622.

[27] Yu, J. S., Oscillation of odd order neutral equations with an "integrally small" coefficients, Chinese Ann. Math. Ser. A 16 (1995), 33-42.

[28] Yu, J. S., Wang, Z. C., Chuanxi, Q., Oscillation of neutral equations, Bull. Austral. Math. Soc. 145 (1992), 195-120

[29] Yu, J. S., Chen, M. P., Zhang, H., Oscillation and nonoscillation in neutral equations with integrable coefficients, Comput. Math. Appl. 35(6) (1998), 65-71.

[30] Yu, J. S., Yan, Jurang, Oscillation in first order neutral differential equations with "integrally small" coefficients, J. Math. Anal. Appl. 187(2) (1994), 361-370.

[31] Yu, J. S., Chen, M. P., Oscillation in neutral equations with an "integrally small" coefficient. Internat, J. Math. Math. Sci. 17(2) (1994), 361-368.

[32] Zahariev, A. I., Bainov, D. D., Oscillating properties of the solution of a class of neutral type functional differential equations, Bull. Austral. Math. Soc. 22 (1980), 365-372.

[33] Zhang, B. G., Oscillation of first order neutral functional differential equations, J. Math. Anal. Appl. 139 (1989), 311-318.

[34] Zhang, G., Gao, Y., Nonexistence and existence of eventually positive solutions for neutral delay differential equations (in Chinese), J. Nonlinear Dynam. Sci. Tech. 5 (1998), 334-335.

S. H. SAKER

FACUlty of Mathematics

AND COMPuter SCIEnCE

ADAM MiCKIEWICZ University

MATEJKI 48/49

60-769 Poznań, Poland

E-MAIL: SHSAKER@AMU.EDU.PL

PERMANENT ADDRESS:

MATHEMATICS DePARTMENT

FACULTy OF SCIENCE

Mansoura UnIVERsity

MANSOURA, 35516, EGYPT

E-MAIL: SHSAKER@MANS.EDU.EG
I. KUBIACZYK

Faculty of Mathematics And Computer Science

AdAm MickiewiCz University

MATEJKI 48/49

60-769 Poznań, POLAND

E-MAIL: KUBA@AMU.EDU.PL 